\title{
Index nominum
}

Abdera 34, $180 \mathrm{f}$.

Abderiten 34f., 180-182

Aelius Dionysius von Halikarnassos 52

Agaue 36, 179, 241f., 244, 246-248, 251253, 376, $402 \mathrm{f} ., 406$

Aischines $16,18-20,27$

Aischylos 5, 17, 28f., 34, 37, 45f., 48-50, 52f., 91, 94-96, 98, 100-105, 107-109, $111,118,125,128,138,154,156,168 \mathrm{f} .$, 178, 194-204, 206, 209, 213, 234, 260, 278, 286, 297, 315f., 319, 321, 352, 371, 378, 381, 384

- Agamemnon 138, 200

- Eumeniden 234

- Perser 45

- Prometheus 45, 200

- Septem 45, 124

Aktaion 251-253

Alexandria $14 \mathrm{f} ., 30,34,135,157,160,200$, $259,264,269-271,295,315,327,335-$ 337, 344, 346, 351f., 354-359, 362, $381 \mathrm{f}$.

Alexandriner $44,138,157-160,162 \mathrm{f}$.

Ammonios Hermeiou 315

Anaxagoras $103,125-128,260,263,301$, $310,315,317,326,351,354,358$

Antiattizist 145-147

Aphareus 21, 26

Apollinarios von Laodikeia $367 \mathrm{f}$.

Apollodor 120

Apollon 75, 188, 234, 236f., 321, 325

Apollonios Dyskolos 91f., $105 \mathrm{f}$.

Apollonios Rhodios 126f., 395, 397

Apollonios Sophistes 91, 97, $104 \mathrm{f}$.

Apostolios, Michael 122, 231 Anm. 35

Aratos 353, 361

Archelaos (Schauspieler) 34f., 127, 180

Argos 35, 154, 216, 235, 237

Aristarch 52, 91, 101f., 318

Aristias 94, 107

Aristobulos 260, 351, 359-362

Aristodemos (Schauspieler) 19

Aristophanes 1, 13f., 17, 34, 48, 53, 64, 95, 97, 103, 111, 118, $120 \mathrm{f.,} \mathrm{139f.,} \mathrm{144,} 193 \mathrm{f.,}$ 199f., 268, 270, 301f., 319, 353f., 358

Aristophanes von Byzanz 4, 64 f., 91f., 168
Aristoteles 22f., 30f., 38, 82, 95, 169, 211, 227-229, 307f., 318, 325, 327

- Poetik 38, 169, 211, 227, $307 \mathrm{f}$.

- Rhetorik 22f., 26, 95, 228

Astydamas 22, 27

Athen 13-17, 22f., 30, 32, 34, 71-73, 121, 139 f., 158, 167, 188 f., 197f., 207, 213, 226, 235, 244, 264, 285, 301, 310, 335, 337, 381, 383

Athenaios 5, 180, 193-204, 264, 268, 312, 337

Athenagoras 352, $354 \mathrm{f} ., 360 \mathrm{f} ., 365$

Athene 71-73, 187-189

Athenodoros (Schauspieler) 21

Autonoe 239, 242-244, 248, 251-253

(5.) Bekkersches Lexikon $15 \mathrm{f} ., 21$

Caligula 269

Charikleia 206-218

Choiroboskos, Georgios 91, 108-111

Christus patiens $1,7,53,120,365,371,384$, 386, 391- 414

Chrysippos 267, 277, 281, 297, 302f., 311f., 353f., 359, 362, 395

Cicero 181, 264, 292

Clemens von Alexandria $6 \mathrm{f} ., 229,231,314$, 335-349, 351f., 354-365

Constantius II. 230

Damis 182,188

Demetrios von Phaleron 34

Demokrit $128,310,315$

Demosthenes $2,16,18-20,27,101,103,120$, 189, 265, 295, 368

Didymos Chalkenteros 52, 101, 103, 136-138, 140,147

Dikaiarchos 65, 67

Diogenes 166f., 185, 269, 303, 353, 376

Diogenes von Apollonia 128

Diogenes Laertios 126, 270, 301f., 310 f., 314, 376

Diogenian 138

Dion Chrysostomos 4f., 153-175, 264, 268, 368 
Dionysios Thrax 103

Dionysos 14f., 20, 78, 99, 157, 226, 239-253, $319,340-346,356,384,403,405 \mathrm{f}$.

Elektra $\quad 78,125,168,188,235,326$

Epicharmos 268

Epikur 261, 286, 310, 312-315, 353

Epiktet 6, 264, 270, 302-309, 312, 327-329, 383

Erasmus von Rotterdam 43

Eros 80, 180, 209, 394-397

Eteokles 74, 124, 166, 213, 231, 292f., $295 \mathrm{f}$., $305,325,364$

Eupolis 194, $199 \mathrm{f}$.

Euripides $1-8,13-21,24-27,29-38$, $43-50,52-55,57$ f., $63-68,74,79,85$, $91-96,98-112,117-130,135-148,153-$ 161, 163-172, 177-191, 193-199, 201206, 209-218, 225-237, 239-251, 253, $259-266,269-271,275-279,281-299$, 301-303, 305f., 309-319, 321f., $324-$ $329,335-341,343-346,351-365,367-$ 370, 372-382, 384f., 387, 391-393, 395, 398, 412; Euripides, der „Bühnenphilosoph“ 6, 263, 285f., 301f., 358; Euripides, der „Tragiker“ 2, 6, 123, 186, 325; Gesamtzahl der Dramen 13, 43-62; Aufführungen im 3. Jh. v. Chr. 31f.; Wiederaufführung von Dramen 15 f., 20-23; Euripides im Schulunterricht 1, 7 f., 33, 177, 205, 217; kaiserzeitliche Auswahlausgabe 4, 47-53, 225, 229; Siege zu Lebzeiten 2, 13

- Aigeus 55, 337

- Aiolos 34, 67, 202, 284, 288, 312 f., 318

- Alexandros 108, 337

- Alkestis 43, 45, 47, 52, 54 f., 65, 78, 82, 146, $190,206,212,225,233,305,325,337$, 356, 384

- Andromache 13, 43, 45, 47, 49, 52, 54, 63, $65,72-79,84-86,121$ f., 142, 144 f., 180, 202, 206, 225, 232

- Andromeda 14, 34 f., 55, 122 f., 180 f., 207, $216 \mathrm{f} ., 230$

- Antigone 337, 339

- Antiope 30, 55, 104, 155, 230, 271, 309, 326, 337, 379

- Archelaos 13f., 32, 127, 141

- Auge 21, 101, 106, 269 f., 337

- Bakchen 2, 5-7, 13, 36, 38, 43, 45 f., 49, 51, 53f., 64 f., 78 f., 157-160, 177, 179, 190,
202, $225-227,229,232,239$ f., 242, 246, 248, 250f., 253, 284, 286, 289, 335, 337, $340-345,371,373,376,378,382,384$, 391-393, 397, 402 f., 405 f., 412 f.

- Bellerophon 56, 80-85, 181, 291, 309, 359

- Chrysippos 259-263, 265f., 310, 337

- Danae 56, 313, 355

- Diktys 102

- Elektra 43, 45, 47, 54, 56, 108, 165, $167 \mathrm{f}$., 284, 286

- Erechtheus 16 f., 118, 141, 283, 337

- Hekabe 20, 43, 45, 47, 49, 51f., 54-56, 65, $128-130,161,185,225,232,265$ f., 283, 337

- Helena 43, 45, 54, 56, 127, 138, 190, 212, 278

- Herakles 32, 36-38, 43, 45, 54, 65, $159 \mathrm{f}$., $163,185,189,232,284$

- Herakliden 43, 45, 65, 226, 232

- Hiketiden 43, 45, 50, 54, 82, 297, $310 \mathrm{f}$.

- Hippolytos 5, 27, 38, 43, 45, 47, 49, 51 f., 54, 56, 65, 74, 78f., 83, 117, 124f., 127, 135, 202, 206, $212 \mathrm{f} ., 215 \mathrm{f} ., 218,225,228 \mathrm{f}$, 232, 284, 289, 294, 303, 315, 322, $337-$ 339

- Hypsipyle 98f., 284, $309 \mathrm{f}$., 337

- Ion $30,38,43,45,50,54,65,82,102,123$, $139 \mathrm{f} ., 146,201,212,291,337,355,380$

- Iphigenie in Aulis 2, 13, 43, 45, 54, 205 , 211f., 214, 217, 315, 337

- Iphigenie bei den Taurern 43, 45, 54, 65 , 191, 217, 337

- Kresphontes 19, 49, 215, 284, 306, 314, 337

- Kreter 49

- Kreterinnen 19, 56

- Kyklops 43, 45, 54, 65, 159, 202, 284, 355

- Medea 7, 26 f., 32, 43, 45, 47, 49, 51f., 54, 64 f., 74, 78 f., 82, 85, 117, 124 f., 131, 146, $186 \mathrm{f} ., 190,198,206,225,233,284,286 \mathrm{f}$. , 290, 302-305, 327 f., 337, 353, 376, 382 f., 391-399, 401-404, 406, 411-413; 2. Fassung $26 \mathrm{f}$.; Kindstötung auf offener Bühne? 27; Medea in der Mittleren Komödie 26

- Melanippe 30, 56 f., 202, 275 f., 282, 284 , 316

- Meleager 56

- Oidipus 50,102

- Oineus 30, 337

- Oinomaos 337, 357 
- Orestes 4, 20 f., 24, 26, 32 f., 38, 43, 45, 47, 49, 51f., 54, 56, 64 f., 78f., 110, 117, 121, $125 \mathrm{f} ., 130,146 \mathrm{f} ., 156 \mathrm{f} ., 166,182,188,191$, 206, 225, 227, 229, 232, 234 f., 284, 290 f., 315, 325, $337 \mathrm{f} ., 355 \mathrm{f} ., 378 \mathrm{f}$.

- Peleus 56

- Peliaden 21, 229

- Phaethon 50, 123, 126, 284

- Philoktet 5, 56, 156,168-173

- Phoinix 18f., 56, 303, 357

-Phönissen 27-29, 33, 43, 45, 47, 49-52, 54, 56, 64 f., 74, 78-80, 82, 165-167, $212-214,218,225,230-232,234,284$, $286-288,292-295,298,318,321,337$, 363, $382 \mathrm{f}$.

- Phrixos 50, 56, 314

- Polyidos 231, 314

- Protesilaos 56, 337

- Rhesos 7, 27, 43, 45, 53f., 63-66, 68, $70-73,76,78,86,94,225,278,391-$ 393, 402, $406-413$

- Skiron 50

- Stheneboia 18, 63, 66 f., 80-86

- Syleus 6, 45, 50, 105, 259-261, 266-269, 271

- Telephos 49f., 337

- Temeniden 312, 337

- Troerinnen 19, 38, 43-45, 53f., 65, 74, 78, 82, 137, 225, 228f., 231, 283, 294, 296, 311, 316, 323

Eusebios von Caesarea 266f., 335, 351f., $354-356,358-365$

Eustathios von Thessalonike 45

Favorinus 293

Flavius Josephus 359

Galen 30

Giunti (Verlag) 43

Gnesippus 197

Gratian (Kaiser) 230

Gregor von Korinth 80

Gregor von Nazianz 7, 367-389, 391

Grigoropulos, Johannes 43

Harpokration 135, $139 \mathrm{f}$.

Hekabe 128, 228, 283, 294, 316f., 382

Helena 24, 26, 129, 228, 316

Heliodor 5, 205-224

Hera $\quad 66,165,188 f ., 325,327$
Herakles 159f., 163, 183f., 189, 206, 303, 353

Hermeias 315

Hermes $131,189,267,269,356,368,383$

Hermogenes $45,66,80,83,323$

Herodian 91f., 94, 96, 100, 103, 106-108, $110 \mathrm{f.,} 128,200$

Herodot 101f., 127, 166, 205, 209, 314

Hesiod 34, 45, 124, 246, 260 f., 263, 309, $318-321,352$ f., 368, 371f., 375, 383

- Opera et dies 45

- Theogonie 124, 353

Hesych 103, 136-140

Hierokles 121, 326-328

Himerios 5, 225f., 232

Homer $2,14,16,18,34,37,47-49,65,105$, 117, 120, 128, 131, 140 f., 153f., 165, 167, $169,178,182,185,188,196,209,211$, $226,234,253,260,277,287,292,309-$ 311, 316, 318-322, 335f., 351-357, 367f., $371 \mathrm{f} ., 375,383 \mathrm{f}$.

- llias 65, 71

- Odyssee 65, 128f., 167, 211, 318

Hydaspes 207f., 212, 217

Hypereides 16

Iamblich 314f., 322, 326

lokaste 27, 74, 165-167, 206, 213f., 288, 293, 296

Ion von Chios 107f., 197

Iphigenie 212, 214

Isaios 16

Ixion 109, 200, 292

Jason von Tralleis (Schauspieler) 36

Jesus Christus 336, 346, 384, 391-414

Johannes (Apostel) 392, 398, 401, 405 f., 408, $410-412$

Johannes Logothetes 80

Johannes Philoponos 106

Joseph von Arimathia 398

Julian (Kaiser) 226, 231, 368f., 386

Kadmos $77,227,242,244,247,250,342 \mathrm{f}$.

Kalasiris 207f., 211-215

Kallias (Komödiendichter) $197 \mathrm{f}$.

Kallimachos 65, 239, 252f., 371

Karkinos $26 \mathrm{f}$.

Katrarios, Johannes 46

Kelsos 336, 354, 364,

Kithairon 244, 252, 307, 342, 345 
Klytaimnestra 156, 235-237

Konstantinopel $\quad 45,52,108,225 f$., 378

Laskaris, lanos 43

Libanios 5, 37, 225 f., 232-237

Lukrez 263

Lukian 5, 34 f., 124, 172, $177-192,227$

Lykinos 124,180

Lykurg 16-18, 20; Lykurgische Verordnung $23,29 \mathrm{f}$

Lysias 16, $139 \mathrm{f.,} \mathrm{297,} 368$

Lysimachos 180

Manutius, Aldus 4, 43

Maria 391-414

Maria Magdalena $\quad 392,408 \mathrm{f}$.

Mark Aurel 6, 307-310, 329

Medea $26 f ., ~ 74,186 f ., 206,233,302-305$, $327-329,376,383,391-399,401-404$, 406, $411-413$

Megara 189

Menander 1, 20, 37, 64, 123, 129, $160 \mathrm{f.,} 163 \mathrm{f}$, $169,189,193,211,227,287,309$ f., 315, 317, 319, 353, 359, 362, $367 \mathrm{f}$.

Menipp 180, 183-186

Moses 261

Musuros, Markos 43

Neoptolemos von Skyros (Schauspieler) 20 Nero 36 f., 154, 156f., 160, 306

Nietzsche, Friedrich $\quad 302,384$

Nil 127

Nonnos 5, 241-255

Ödipus $\quad$ 5, $27 \mathrm{f.,} \mathrm{30,} \mathrm{36,} \mathrm{154,} \mathrm{157,} \mathrm{214,} \mathrm{309,}$ 378,382

Odysseus $71,73,128,169-172,184,211$, 236, 303, 317

Olympiodor 314f., 323-326

Orest $\quad 24,32,121,156$ f., 167, 191, 227, 234 $237,291,326,356$

Origenes $336,354,358,364$

Orion $55,135,141-143,281,361$

Orpheus $126 \mathrm{f} ., 184,320,353,376,383$

Ovid 265, 369

Parrhesiades 180, 182f., 185

Parther 36

Pelopiden 154-157
Pentheus $36,77,177,179,226,239-255$, $335,341-346,378 f ., 383,402-406$

Perikles 197, 286

Perseus 102, $180 \mathrm{f} ., 216 \mathrm{f}$.

Persinna 207, 217

Phaidra 5, 212-216, 289, 323

Philochorus 281, $297 \mathrm{f}$.

Philoktet 153, 168-173

Philon von Alexandria 6, 180, 259-274, 336

Philoxenos 91, 97-100,142f.

Photios 138, 226

Phrynichos 107, 143-145

Planudes, Maximos 45

Platon 2, 30, 154, 182 f., 229, 231f., 261, 263, 276f., 286, 289, 295, 297, 299, 305f., 310, $315 \mathrm{f} ., 319-322,324,326 \mathrm{f.}, 329,335 \mathrm{f}$., 339 f., 352f., 358, 365, 373

- Alkibiades I 324-326

- Apologie 305, 315

- Gorgias 30, 325-327

- Ion 30

- Kriton 305

- Phaidon 315, 325

- Phaidros 297, 320

- Politeia 231f., 319-321, 324

- Nomoi 320, 324

- Symposion 30,199

Plotin 316-318, 323f

Plutarch $6,29,36,158,167,263,275-300$ 302, 306, 311, 314, 317, 337, 355, 359, 362 f., 373

Podagra $190 \mathrm{f}$.

Pollux, Julius $\quad 145-147,201,229$

Polyneikes 27f., 165, 167, 213, 289, 293f., $305 f ., 325,364,375,380$

Polyxena 260, $265 \mathrm{f}$.

Porphyrios $130 \mathrm{f} ., 318 \mathrm{f}$.

Proklos 6, 316f., 319-325

Prometheus $188 \mathrm{f}$

Ps.-Hekataios 351, 359-362, 365

Pylades (Pantomime) 38, 130, 229

Seneca 1, 177, 216, 369

Sextus Empiricus $\quad 6,263,301,306,310-315$, 329, 356

Simplikios 327-329

Sokrates 270f., 295, 301-303, 305, 309, 317, $321-323,325-327,329,351,353 f ., 358$, 383 
Sophokles 2, $4 \mathrm{f} ., 27-29,37,45,47-50,52 \mathrm{f}$, 64, 91, 94-98, 100-111, 118, 128, 146, $154-156,165,168,178,194-204,206$, $209,211,213,216,227,232,260,278$, 286, 294, 303, 309, 315f., 321, 352 f., 358 360, 371, 373, 378f., 381, 384

- Aias 45, 47, 105, 109f., 325, 371,

- Antigone 45, 213, 371

- Elektra 45, 47, 156

- König Ödipus 45, 47, 197, 303, 371, 378

- Ödipus auf Kolonos 45

- Oinomaos 19, 105

- Trachinierinnen 45

Spartaner $121 \mathrm{f}$.

Stephanos von Byzanz $139 \mathrm{f}$.

Stobaios, Johannes $53-55,57,120,129 \mathrm{f}$., 201f., 269, 271, 281, 310, 314 f., 328, 356, $361 \mathrm{f}$.

Synesios $315,328,369,379$

\section{Tatian 352f., 356}

Teiresias 77, 242, $244 \mathrm{f}$., 250, 252f., 298, 335, $341-343,346,356,405,413$

Theagenes 206-210, 212-215, 218

Theben $16,27,154,189,213,226,241-243$, 246, 250 f., 253, 306, 340-343, 379, 383

Themison (Komponist) 37

Themistios 5, 225, 227-232, 315

Theodektas 27

Theodoret 351f., 355-357, 359, 362-365

Theodorides 26

Theodoros Prodromos 45

Theodoros (Schauspieler) 19

Theodosios von Alexandria 108-110

Theodosius I. $227 \mathrm{f} ., 230$

Theokritos 45, 123

Theon von Alexandria $101 \mathrm{f}$.

Theophilos von Antiochia 341, 352 f., $355-$ 357, $361 \mathrm{f}$.

Theophrastos 319

Theseus 72, 74, 213, 228, 297

Thettalos (Schauspieler) 21

Thomas Magister 5, 65, 239, 253, 370, 384

Thukydides 118, 198, 353, 368

Thyamis $212 \mathrm{f}$.

Timokles 27, 182, 188

Triklines, Nikolaos 46

Triklinios, Demetrios $\quad 44-47,119,177,179$

Troja 75f., 129, 137, 154f., 170, 236, 317, 337, 353, 407, 409, 411
Tryphon von Alexandrien 100

Tyndareos $235 \mathrm{f}$.

Tyrtaios 18, 320

Tzetzes, Johannes $\quad 45,118,122$

Victorius, Petrus (Pier Vettori) 43

Vitruv 263

Xenophon 16, 205, 213, 263, 286

Zeus 137, 159, 188f., 228, $250 \mathrm{f} ., 263,268$, $280,296,304,306,310-312,316,319$, 356,382 
